\title{
Relationship Between IBICC Imaging And SEU In CMOS ICs*
}

\author{
F. W. Sexton, K. M. Horn, B. L. Doyle, J. S. Laird ${ }^{+}$M. Cholewa ${ }^{+}$, A.Saint ${ }^{+}$, and G. J. F. Legge ${ }^{+}$ \\ Sandia National Laboratories, Albuquerque, NM 87185-5800
}

\begin{abstract}
lon-beam-induced charge-collection imaging (IBICC) has been used to study the SEU mechanisms of the Sandia TA670 16K-bit SRAM. Quantitative charge-collection spectra from known regions of the memory cell have been derived with this technique. For $2.4-\mathrm{MeV} \mathrm{He}$ ions at normal incidence, charge collection depth for a reverse-biased $\mathrm{p}+$ drain strike is estimated to be $4.8 \pm 0.4 \mu \mathrm{m}$. Heavy-ion strikes to the reverse-biased $\mathrm{p}$-well result in nearly complete collection of deposited charge to a depth of $5.5 \pm 0.5 \mu \mathrm{m}$. A charge amplification effect in the $n$-on drain is identified and is due to either bipolar amplification or a shunt effect in the parasitic vertical npn bipolar transistor associated with the $n+/ n^{-}$substrate, $p$-well, and $n+$ drain. This effect is present only when the $n+$ drain is at $0 \mathrm{~V}$ bias. When coupled with previous SEU-imaging, these results strongly suggest that the dominant SEU mechanism in this SRAM is a heavy-ion strike to the n-on transistor drain.
\end{abstract}

\section{Introduction}

Single-event-upset in integrated circuits (ICs) is a complex function of circuit design and the physics of charge collection in the internal structure of the IC. Typically, error cross sections are measured by broad-area chip exposures in heavy-ion accelerators. This measures the cumulative response of all sensitive regions of the chip. It is well known, however, that different regions of an IC can have different upset thresholds. Without specialized test circuits or robust modeling capability, it is difficult, if not impossible, to unambiguously separate the contribution to upset from discrete regions or circuit elements of the IC. If one wishes to mitigate SEU with either technology or design fixes, it is essential that one can directly identify the upset locations and understand the upset mechanisms. With SEU imaging, we can resolve those regions of an integrated circuit which are susceptible to upset by energetic heavy ions [1-3]. This technique does not, however, yield information on the underlying charge collection processes responsible for upset. Conversely, ion-beam-induced charge-collection (IBICC) imaging can directly image the charge collection regions of an integrated circuit [4,5], but does not identify those areas that are sensitive to heavy-ion

* This work was supported by the U.S. Department of Energy under contract number DE-AC04-76DP00789.

+ Micro Analytical Research Centre, University of Melbourne, Parkville, VIC, Australia 3052 strikes, since imaging is performed at energies below the upset threshold.

A primary goal of this work is to understand IBICC imaging from the perspective of single-event upset, and determine whether the technique can improve our understanding of SEU mechanisms. We will present IBICC images of the Sandia TA670 16K-bit CMOS SRAM measured with a charge-sensitive preamp connected to either $\mathrm{V}_{\mathrm{DD}}$ or $\mathrm{V}_{\mathrm{Ss}}$. The 2-D images derived from this technique are similar to those obtained by Breese et al. [6] on the Oxford microbeam. We then present a detailed analysis of these images and explain the position dependence of the charge- collection signal magnitude. Additionally, we explain why some regions cannot be imaged at all, due to the inherent design of the IC. Then, we present charge-collection depths calculated from spectra of various regions of the IBICC image using the approach of McNulty et al. $[7,8]$. Finally, we interpret the IBICC results in connection with previous SEU-imaging that showed the n-drain to be most sensitive to upset.

\section{Experimental Details}

Charge-collection imaging of integrated circuits is accomplished by using the IC as a detector. A charge-sensitive preamp is connected to either power bus, $\mathrm{V}_{\mathrm{DD}}$ or $\mathrm{V}_{\mathrm{sS}}$, and the resulting signal is amplified and fed into a multi-channel analyzer. For the charge collection images presented here we used the microbeam facility at the Micro Analytical Research Center at the University of Melbourne where spot sizes of approximately 0.05 micron have been obtained. A $2.4 \mathrm{MeV}$ helium beam at 1500 particles/second was used for the charge collection images in this work. Typical spot size was approximately $0.1 \mu \mathrm{m}$. Please note that the LET for this ion $\left(\sim 1.0 \mathrm{MeV}-\mathrm{cm}^{2} / \mathrm{mg}\right.$ at the silicon surface) is below that required to upset this device $(\sim 18$ $\mathrm{MeV}-\mathrm{cm}^{2} / \mathrm{mg}$ ). Penetration depth is about $8.75 \mu \mathrm{m}$. Charge-collection data was stored in a $256 \times 256$ array of pixels. Each pixel has a charge-collection spectrum associated with it. The median of the charge-collection spectrum at each pixel is calculated and a false-color scale is applied to generate the IBICC images shown in the next section.

The test device used in this work was the TA670 16K-bit SRAM. This integrated circuit is a $2-\mu \mathrm{m}$ design that was fabricated at AT\&T Bell Laboratories in their $1.25-\mu \mathrm{m}$ radiation-hardened technology [9]. A twin-tub process 
fabricated on 2- $\mu \mathrm{m} n$-on- $n$ epitaxial silicon forms the basis of this CMOS technology. One level of polysilicon and two levels of metal are used for interconnections in this design. A 6-transistor design was used for the memory cell of the TA670. Although the memory-cell design includes provision for feedback resistors for SEU hardening, no feedback resistors were included in the memory cell of the TA670s in this study. Device temperature during measurements was at ambient, about $30^{\circ} \mathrm{C}$

\section{IBICC Imaging}

IBICC images were taken with two different configurations. In the first series of measurements, the charge sensitive preamp was connected to $V_{s s}$ while $V_{D D}$ was held at ground. Input bias to the preamp was varied from 0 to $-15 \mathrm{~V}$ in steps of $5 \mathrm{~V}$, giving an effeclive bias of 0 ro $+15 \mathrm{~V}$ for $V_{D D}$ relative to $V_{S S}$. All other imputs to the device were held at ground. In the second configuration, the charge sensitive preamp was connected to $V_{D D}$ while $V_{S S}$ was connected directly to ground. Brases from 0 to $15 \mathrm{~V}$, in $5 \mathrm{~V}$ steps, were applied to $\mathrm{V}_{D B}$ through the preamp. All other inputs were held at ground.

In Figure la we show an IBICC image measured at $V_{S s}$ with $-15 \mathrm{~V}$ bias. In Figure $1 \mathrm{~b}$ we show the design layout of the same region of the TA670. The large purple region in Figure la clearly corresponds to the p-well region of the TA670. The smaller features within the p-well correspond to the $\mathrm{n}+$ diffusions that form the $\mathrm{n}$-channel transistor

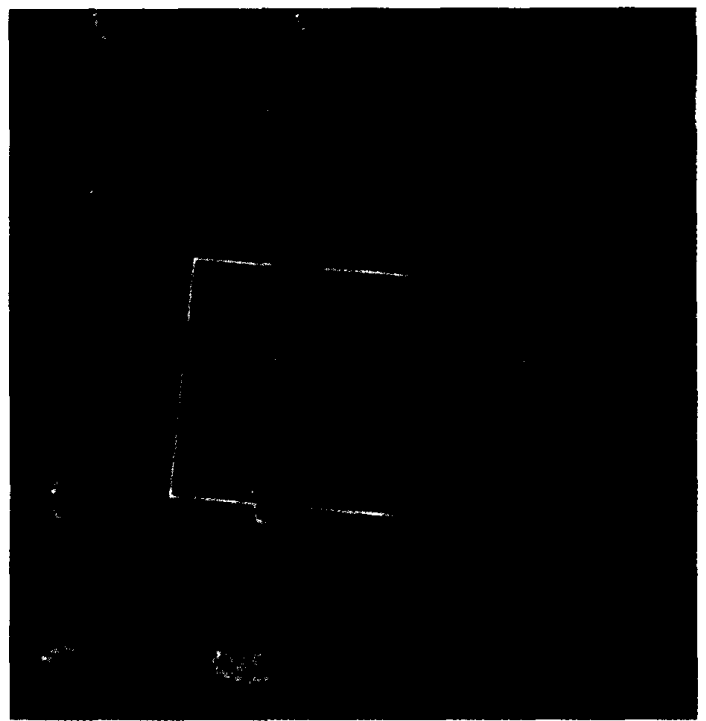

a) sources and drains. The large black region in Figures 1a) and $b$ ) corresponds to the n-well of the TA670, and the red fearures within correspond to the $p+$ diffusions that form the p-channel transistor sources and drains. The area corresponding to a single memory cell is highlighted in both a) and $b$ ) and the $n$ - and p-channel sources and drains are labeled in $b$ ). Note that the $n$ - and $p$-channel sources and the pass transistor drains are bisected by the cell outline. The memory cell is repeated in the array by mirroning in both the $\mathrm{x}$ and $\mathrm{y}$ direction. The $\mathrm{L}$-shaped $\mathrm{n}+$ diffusions are the $n$-channel drains, and the smaller $p+$ diffusions form the $\mathrm{p}$-channel drains. Each $\mathrm{n}$-drain is electrically connected to the p-drain nearest to it by a metal line (not shown in the figure). Note in Figure la that the $n+$ sources can be imaged. while none of the $p+$ sources can be imaged. The $n+$ and $p+$ drains are maged in pars; that is, the only $p+$ drains that can be seen are those that are connected to $\mathrm{n+}$ drans that can also be imaged

Figure 2 shows an IBICC images of the TA670 taken at a blas of 0 and $-5 \mathrm{~V}$ from the $V_{\text {ss }}$ pin. A scale relating color io charge amplitude is shown on the right. Please note that some adjustment in pulse-height baseline may have been made between different biases, so this scale should be used for relative. not absolute. intensity. At OV bias (Figure 2a), all $n+$ source and drain regions can be imaged. with the sources showing more charge collection. No structure is evident in the $n$-well region. Al a bias of $-5 \mathrm{~V}$ (Figure $2 \mathrm{~b}$ ) the p-well region shows definite structure at the edges, and the $\mathrm{n}+$ sources and drains within are well defined. Also, some of

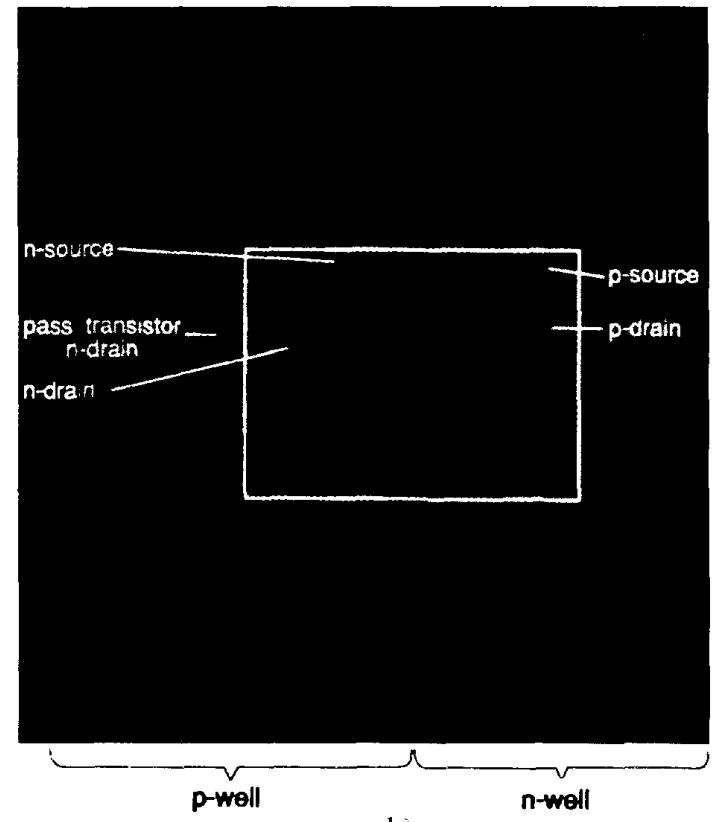

b)

Figure 1: The IBICC image a) measuled at $V_{s y}$ with $15 \mathrm{~V}$ bras is compared to the design layout b) of the TA670. The black region corre sponds to the $\mathrm{n}$-well, while the purple regron corresponds to the p-well 
the drains that were imaged in the p-well at $0 \mathrm{~V}$ have disappeared at $-5 \mathrm{~V}$ bias. The $\mathrm{n}+$ sources and drains in the p-well exhibit a center of high charge collection, surrounded by an annulus of lower charge collection (the dark green regrons). The image at $-15 \mathrm{~V}$ (Figure $1 \mathrm{a}$ ) exhibits the same structure. Images at -10 (not shown) and $-15 \mathrm{~V}$ (Figure $1 \mathrm{a}$ ) bias have the same structure and differ only in magnitude of charge
IBICC images taken in the second configuration measuring charge trom $V_{D D}$ are shown in Figure 3. At $0 \mathrm{~V}$ bias (Figure 3a), we can distinguish the p-well, but the $n+$ sources and drains are not well defined compared to Figure 2a. Also, the $p+$ drains within the n-well are not well defined. At $+15 \mathrm{~V}$ bias, the IBICC image shows similar structure as at $-15 \mathrm{~V}$ in the $\mathrm{V}_{\mathrm{ss}}$ configuration, but the image is not as well defined

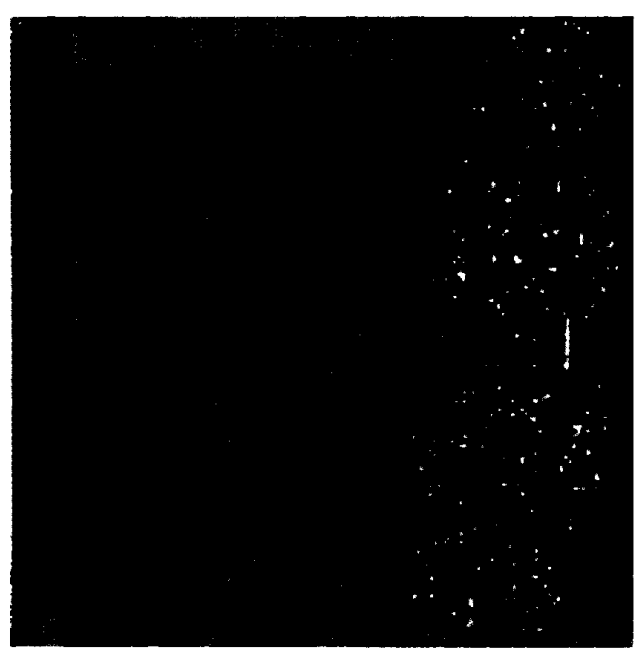

a)
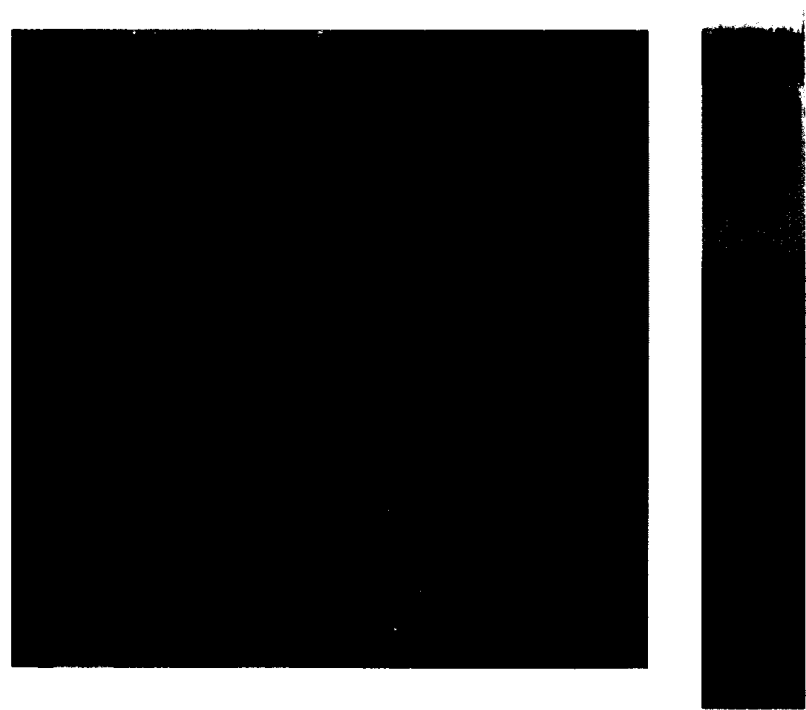

200

b)

Figure 2: The IBICC image measured at $V_{s s}$ with a) $0 \mathrm{~V}$ bias and b) $5 \mathrm{~V}$ buas. The color scale at right 15 in arbitiary units, with white ie terring to maximum charge and black to munimum charge

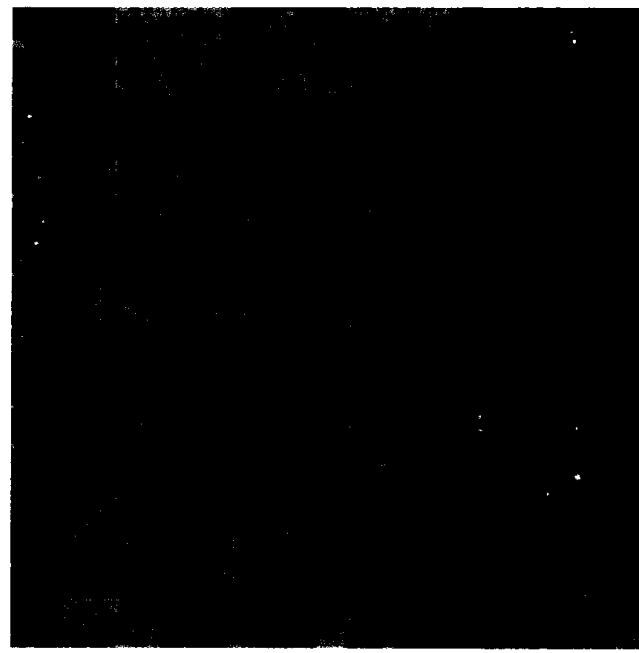

a)

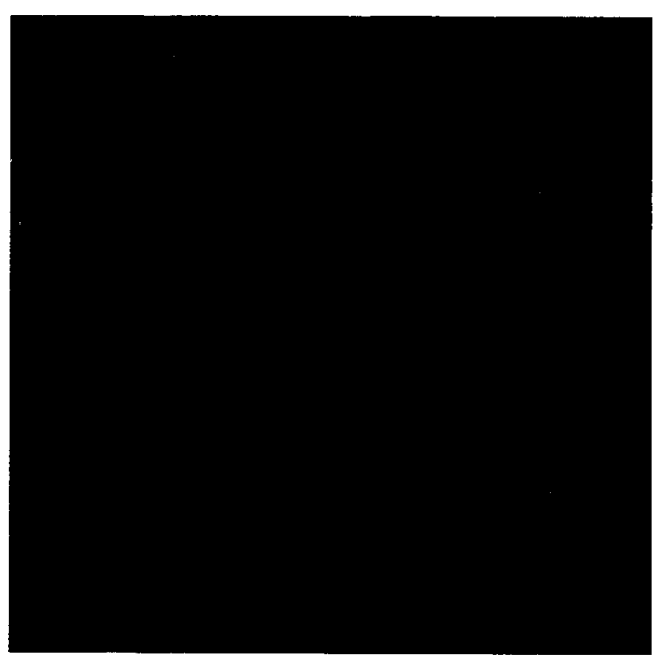

b)

Figure 3: The IBICC umage measured at $V_{D D}$, with a) $0 \mathrm{~V}$ bras ard b) $+15 \mathrm{~V}$ bias 


\section{Charge-Collection Spectra}

As stated earlier, each pixel of an IBICC image corresponds to the median of a charge-collection spectrum. In order to quantify the charge collected at any pixel the gain of the charge-sensitive preamp and shaping amplifier must be calibrated with a known energy peak. The energy scale for a second series of IBICC images was calibrated by measuring a Rutherford Backscattering (RBS) spectrum of a 2.4-MeV He beam directed onto a microscope-slide cover glass. This spectrum has two well defined edges at 0.88 and $1.4 \mathrm{MeV}$ corresponding to a $\mathrm{He}$ ion scattered off an $\mathrm{O}$ and $\mathrm{Si}$ atom, respectively.

The composite charge-collection spectrum from the IBICC image of Figure 1a is shown in Figure 4. This is similar to charge-collection spectra obtained by McNulty et al. [7]. This and subsequent charge-collection spectra are analogous to the output of a multi-channel analyzer used in nuclear electronics, where the $\mathrm{x}$-axis is deposited energy (or collected charge) and the $y$-axis is the number of counts measured per channel. A well-defined peak is evident at 1.4 $\mathrm{MeV}$, and significant counts are registered at energies up to 3.8 $\mathrm{MeV}$, more than twice the incoming energy of the $\mathrm{He}$ ion beam. The area under each peak is related to the spatial area of specific regions of the IC. Because the p-well region is the largest feature on the IC, charge-collection in this region gives the largest peak. This spectrum was derived from an uncalibrated IBICC scan. The energy scale has been normalized to the 1.4-MeV p-well peak of the calibrated scan shown in Figure 5 below.

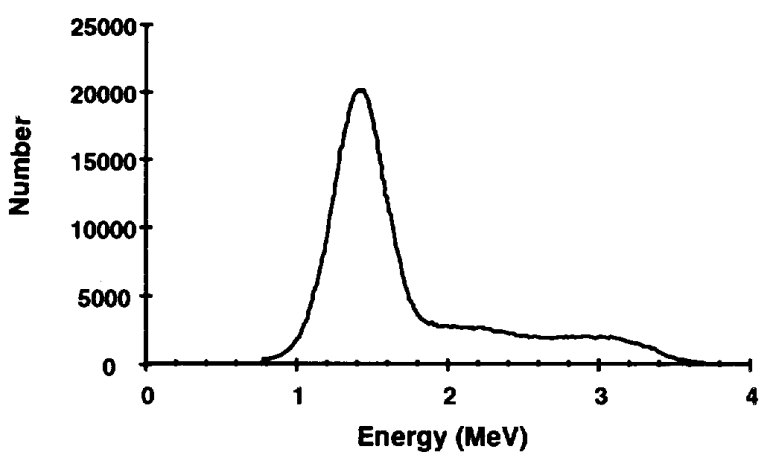

Figure 4: A composite charge-collection spectrum for all regions of the IBICC image of Figure 1a. A well-defined peak due to heavyion strikes at the p-well is evident. The high-energy tail above 2 $\mathrm{MeV}$ is associated with heavy-ion strikes to the n-source/drain regions.

The calibrated charge-collection spectra from the n-well, $p$-well and $n+$ source and drain are shown in Figure 5. The p-well spectrum has a pronounced peak at $1.41 \mathrm{MeV}$, while the $n+$ source and drain region has a well-defined peak at $1.65 \mathrm{MeV}$ and a broad high-energy tail to above $3 \mathrm{MeV}$. The background noise level can be determined from the n-well region where no pn-junctions are intersected. No structure is evident in the $n$-well spectrum, as expected. The second peak in the p-well spectrum at $2.14 \mathrm{MeV}$ is discussed below.

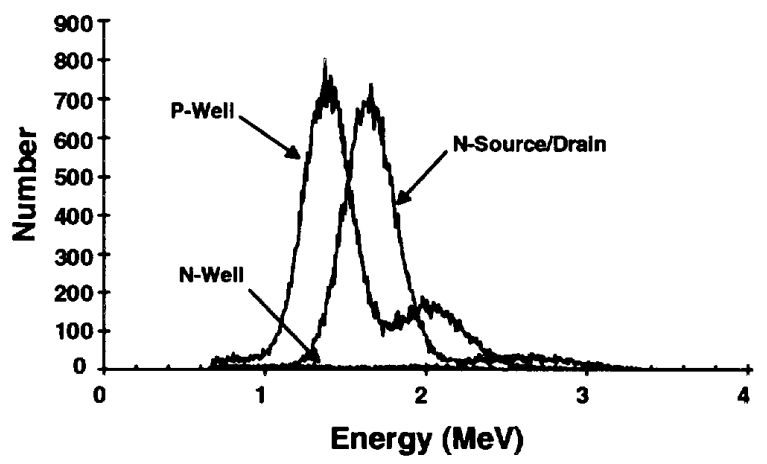

Figure 5: Charge-collection spectra derived from various regions of the IBICC image of Figure la.

The charge-collection spectrum of the $p+$ drain region is compared to the p-well spectrum in Figure 6. This spectra is from an uncalibrated IBICC image, so the energy scale has been normalized to the p-well peak at $1.4 \mathrm{MeV}$ of Figure 5 . The $\mathrm{p}+$ drain peak at $\sim 1.25 \mathrm{MeV}$ is very close to the $\mathrm{p}$-well peak at $1.4 \mathrm{MeV}$. Note also that the p-well spectrum is single-valued with a maximum of about $1.9 \mathrm{MeV}$. The second peak in the p-well spectrum of Figure 5 is an artifact of the data analysis software. Apparently, part of a nearby $\mathrm{n}$-drain region was included when this spectrum was derived from the IBICC image.

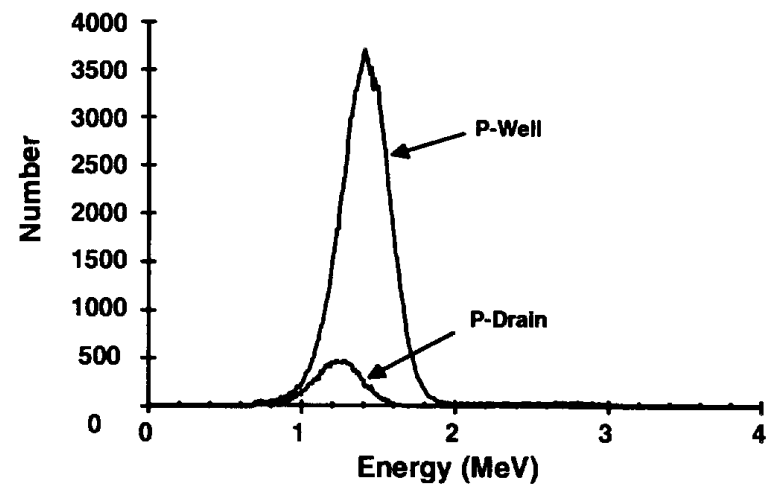

Figure 6: Charge-collection spectra for heavy-ion strikes to a reverse-biased $p+$ drain region. Note that charge-collection in the $\mathrm{p}+$ drain is comparable to the $\mathrm{p}$-well region.

\section{IBICC Analysis}

A cross section of the TA670 technology through an inverter is shown in Figure 7. The p-well and n-well regions are about $1.5 \mu \mathrm{m}$ deep. $\mathrm{N}$-type source and drain regions in 


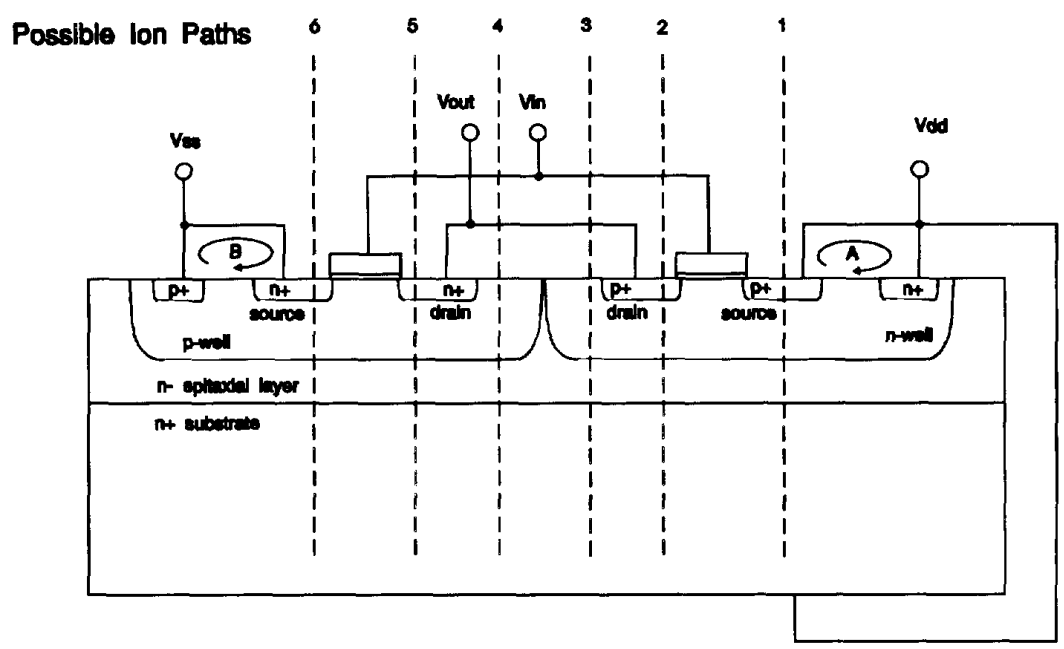

Figure 7: A cross section of the vertical structure of the CMOS inverter forming one half of a memory cell. Several different ion paths through this structure are indicated by the dashed lines. Paths for current resulting from ion strikes to the $\mathrm{p}$ - and $\mathrm{n}$ - sources are labeled "A" and "B," respectively.

the p-well are about $0.3 \mu \mathrm{m}$ deep, while p-type source and drain regions in the $n$-well are about $0.4 \mu \mathrm{m}$ deep. Electrically, the $\mathrm{n}$-well, $\mathrm{n}+$ substrate, and $\mathrm{p}+$ sources are tied to $\mathrm{V}_{\mathrm{DD}}$. The p-well and $\mathrm{n}+$ sources are tied to $\mathrm{V}_{\mathrm{ss}}$. In the memory cell, the gates for the $\mathrm{n}$ - and $\mathrm{p}$-channel transistors are connected together, and the drains are wired together.

We also show in Figure 7 possible ion paths through the various regions of the TA670. A strike along path 3 through the $n$-well region will not be imaged with the preamp connected to either $V_{\mathrm{ss}}$ or $\mathrm{V}_{\mathrm{DD}}$, because there is no $\mathrm{p}$ - $\mathrm{n}$ junction to separate electron-hole pairs generated by passage of the heavy ion. This region is at the noise limit of the preamp. A strike along path 1 through the p-source will not be imaged in either configuration because this $\mathrm{p}-\mathrm{n}$ junction is electrically shorted. Current generated by minority-carrier collection flows through the closed loop indicated by 'A.,' therefore, no path exists for current to either $\mathrm{V}_{\mathrm{Ss}}$ or $\mathrm{V}_{\mathrm{DD}}$.

The p-well region is always connected to $V_{s s}$ while the $n$ - and $n+$ regions below it are connected to $V_{D D}$. When positive bias is applied to $V_{D D}$ relative to $V_{S S}$, this junction will be reverse biased. An ion strike along path 4 will result in charge collection into both $\mathrm{V}_{\mathrm{Ss}}$ and $\mathrm{V}_{\mathrm{DD}}$, and will therefore be imaged in both configurations. This corresponds to the p-well of Figure 1a.

The $n$-sources are electrically shorted to the p-well region and tied directly to $\mathrm{V}_{\mathrm{ss}}$. Since the $\mathrm{n}$-source/p-well junction is at zero bias, current resulting from a strike to this junction along path 6 will see a closed loop path labeled 'B,' and cannot be measured through $\mathrm{V}_{\mathrm{DD}}$ or $\mathrm{V}_{\mathrm{ss}}$. However, path 6 also includes the p-well/n-epi junction. These junctions together form a vertical bipolar npn transistor in a common-emitter mode. Minority-carrier charge can be amplified resulting in a significantly enhanced charge collection $[10,11]$. A shunt effect $[12,13]$ connecting the high potential n-epi/substrate region to the low potential n-source could also explain the enhanced charge collection in this region. In Figure 1a, the largest charge collection occurs in the center of struck $n+$ and $p+$ junctions, contrary to earlier observations by McNulty [7] that there was enhanced collection at junction edges'.

Again we note that the $n+$ and $p+$ drains only appear in pairs. This is consistent with the bi-stable operation of the memory cell. When the common gate of an inverter is at a high potential, the n-channel transistor is "on," electrically connecting the $\mathrm{n}$ - and $\mathrm{p}$-drains to the $\mathrm{n}$-source. Under these conditions a strike along path 5 is equal to path 6 . A strike along path 2 sees a single reverse-biased pn junction, so no amplification mechanism exists to enhance charge collection here. Accordingly, the p+ drain has lower charge collection. On the opposite side of the memory cell, the inverter gate is at a low potential and the $\mathrm{n}$ - and $\mathrm{p}$-drains are electrically connected to the p-source. A strike along path 2 is now equal to 1 and the $\mathrm{p}+$ drain will not be imaged. A strike along path 5 through the $n$-drain sees two reverse-biased junctions, and no amplification mechanism is present. Charge collection in the n-drain regions in the IBICC images can barely be distinguished from $p$-well charge collection. It could be argued that lower charge is collected here because the reverse-biased junctions compete for charge. However, in the $V_{s s}$ IBICC images this explanation errs because $V_{s S}$ is the common anode for both

\footnotetext{
${ }^{1}$ In subsequent communications, McNulty has stated that his data do not support enhanced edge collection.
} 
of these diodes. Minority carriers collected in either of these diodes will result in a current in the $V_{\text {ss }}$ path.

\section{Charge-Collection Depths}

McNulty $[7,8]$ has estimated the charge collection depth in NMOS and CMOS devices based on the assignment of peaks in the charge collection spectra to specific junctions. One weakness of this approach with broad-beam charge-collection spectra is the uncertainty associated with assigning peaks to specific regions of the device. The composite spectrum of Figure 4 clearly illustrates this difficulty. There is no such ambiguity with IBICC imaging, where we can derive charge-collection spectra from known regions of the device.

Following McNulty's approach, we calculated first the energy loss in device overlayers using the TRIM90 code [14], and then calculated the depth required to accumulate a deposited energy equal to the peak value. The TA670 has two layers of oxide totaling $1.8 \mu \mathrm{m}$ and two layers of metal totaling $1.5 \mu \mathrm{m}$ over the $\mathrm{n}+$ and $\mathrm{p}+$ source and drain regions and most of the p-well region. After passing through these layers, the He beam is reduced in energy to $1.6 \pm 0.1 \mathrm{MeV}$.

In Figure 5, the p-well peak is at $1.4 \mathrm{MeV}$. This implies that virtually all charge generated in this region is collected. The 1.4-MeV peak corresponds to a charge collection depth for a p-well strike of $\sim 5.5 \pm 0.5 \mu \mathrm{m}$. This is much deeper than the 1.5- $\mu \mathrm{m}$ thick epitaxial layer (depth after processing) and deeper than first-order funneling theory [15] predicts in the heavily-doped substrate region $(\sim 0.3$ $\mu \mathrm{m})$. This is due in part to the fact that we are measuring both diffusion and drift currents in this work, as discussed below. Since the He ion can deposit a maximum of $1.6 \pm 0.1$ $\mathrm{MeV}$ energy, the energy above this value in the $\mathrm{n}+$-drain spectrum of Figure 5 must be due to an amplification mechanism as discussed in the previous section. The 1.25-MeV peak for the p+ drain spectrum of Figure 6 corresponds to a charge-collection depth of $4.8 \pm 0.4 \mu \mathrm{m}$. Calculated charge-collection depths are summarized in Table 1 below.

\begin{tabular}{|c|c|c||}
\hline Structure & $\begin{array}{c}\text { Peak } \\
(\mathbf{M e V})\end{array}$ & $\begin{array}{c}\text { Charge-collection } \\
\text { depth }\end{array}$ \\
\hline p-well & $1.4 \pm 0.1$ & $5.5 \pm 0.5$ \\
\hline n+ drain & $1.65 \pm 0.1$ & $\begin{array}{c}\text { excess charge } \\
\text { mechanism }\end{array}$ \\
\hline p+ drain & $1.25 \pm 0.1$ & $4.8 \pm 0.4$ \\
\hline
\end{tabular}

Table 1: Spectral peak position and calculated charge-collection depth for TA670 structures.
In these IBICC experiments, we used a charge-sensitive pre-amplifier that has a time constant on the order of microseconds, while the TA670 memory cell has a switching speed of several nanoseconds. Charge measured at long times will therefore include diffusion as well as drift components. These IBICC measurements may therefore include charge that does not normally lead to upset. For this reason, the charge-collection depths calculated here are an upper bound. More sophisticated and faster time-resolved IBICC studies will be necessary to improve these estimates further.

\section{Relation to SEU}

We return now to our initial goal of relating IBICC images to SEU. Typically in CMOS ICs, the "off" $n$ - and p-channel drains are the regions most sensitive to upset, because charge collection is strongest in the reverse-biased diodes. In the absence of an amplification mechanism, much less charge is collected by the zero-biased drains of the "on" $\mathrm{n}$ - and p-channel transistors. Also, excess charge is rapidly removed by the "on" transistor preventing a voltage rise on the struck node. When an amplification mechanism is present, however, sufficient charge could be collected from a zero-biased diode to cause upset if the "on" transistor is not able to remove the excess charge quickly enough.

Previous SEU imaging [2] of the TA670 has shown that the $n$-drains are more sensitive to upset than the p-drains. The IBICC-imaging results of this work combined with this earlier SEU imaging imply that strikes to the n-on drain dominate upset for these devices with no feedback resistors. This is in contradiction with simulations of an earlier $2-\mu \mathrm{m}$ technology by Fu et al. [11] which showed that strikes to the $\mathrm{n}$-on transistor drain would not result in upset because this mechanism is self-limiting. As the $n$-on drain voltage rises to $\mathrm{V}_{\mathrm{DD}}$ from $0 \mathrm{~V}$, the $\mathrm{n}+$ emitter of the vertical npn transistor is reverse biased, shutting off the bipolar amplification mechanism.

The apparent discrepancy between the results of this work and that of Fu's may be due to differences in the structure of the TA670 and the earlier device modeled by Fu. The TA670 used in this work has a narrower base region, and a subsequently larger bipolar gain which could lead to a stronger bipolar effect. A narrower base region would also strengthen a shunt mechanism. Whether either of these mechanisms can lead to upset depends to first order on the structure of the technology and design of the latch circuit. Consequently, an n-on strike mechanism may be present in one design and technology and not another. Three-dimensional transport simulations of $\mathbf{n}$-on strikes are planned to elucidate the bipolar vs shunt mechanism.

There are potential difficulties in extrapolating mechanisms from low-LET ions to high-LET ions closer to 
the upset threshold. For this study, we purposely chose ions with LET below threshold so that the state of the memory cell would be constant throughout the scan. If the memory cell upset during each pass through a sensitive region, the state during charge collection would be ambiguous. Also, the image would depend on the direction of scanning across the cell. The downside of using a low-LET ion, however, is that the strength of the amplification mechanism observed here may be much different with a high-LET ion. For a bipolar mechanism, for example, a high-LET ion will cause more charge injection into the parasitic bipolar base. This could lead to a stronger bipolar effect and more charge collection. Conversely, higher charge injection could lead to a more rapid voltage transient on the emitter (drain) and earlier extinction of the bipolar effect. The result would be less total charge with a higher LET ion and a different upset mechanism dominating. To resolve this problem, it would be better to use an ion with an LET just below upset for IBICC imaging. Future efforts will explore the use of high vs. low LET ions.

Finally, the $p+$ charge-collection depth of $4.8 \mu \mathrm{m}$ agrees well with the estimated charge collection depth for the SA3300 16-bit microprocessor fabricated in this same technology [16]. In this previous work, we estimated the charge collection depth for a p-drain strike to be $4.5 \mu \mathrm{m}$ by matching threshold LET with the critical charge calculated from lumped-parameter circuit modeling. This agreement may be fortuitous, however, since, as discussed above, in these IBICC measurements we are collecting charge for long times relative to the switching time of the memory cell. Also, the modeling performed earlier could not account for the amplification effect observed in this work.

\section{Summary}

The $\mathrm{n}+$ source/drains, $\mathrm{p}$-well, and reverse-biased $\mathrm{p}+$ drains are clearly identified in IBICC images of the TA670 16K-bit SRAM. P-drains were easily imaged in the bias condition sensitive to SEU. However, $n$-drains that were biased-off were not well defined against the signal coming from the underlying p-well. The largest charge-collection signal came from the $\mathrm{n}$-channel drains when biased at $0 \mathrm{~V}$. This results from enhanced charge-collection in the n-on drain region due to either a bipolar effect or shunt effect in the parasitic vertical npn transistor. This result, coupled with earlier SEU-imaging, strongly implies that heavy-ion strikes to the n-on drains are the dominant SEU mechanism for this memory cell with no feedback resistors. We discussed potential difficulties in the interpretation of these results due to extrapolation from the low-LET ion used in these IBICC measurements to the high-LET ion necessary for upset. Charge-collection depths were calculated from quantitative charge-collection spectra derived from known regions of the memory cell. Depths were estimated to be
$4.5 \pm 0.4 \mu \mathrm{m}$ and $5.5 \pm 0.5 \mu \mathrm{m}$ for heavy-ion strikes to the p-off drain and reverse-biased p-well junctions. These numbers are an upper limit to the charge-collection depths associated with SEU. The power of the IBICC technique over broad-beam charge-collection spectra lies in its ability to derive charge-collection spectra from known regions of the IC. In CMOS ICs, charge-collection in the p+ or $n+$ drain regions is masked by the larger $p$-well response in a broad-area spectrum.

\section{Acknowledgements}

The authors would like to thank David Barnes of the Sandia Satellite Technology group for financial support of this work. We also thank Peter Winokur for his encouragement and critical review. Finally, the authors thank the anonymous reviewers of this manuscript for their insightful comments that have clarified much of the discussion of this paper.

\section{References}

[1] K.M. Horn, B.L. Doyle, D.S. Walsh, and F.W. Sexton, "Application of the Nuclear Microprobe to the Imaging of Single Event Upsets in Integrated Circuits," Scanning Microscopy, vol. 5 no. 4, 969 (1992).

[2] K M. Hom, B.L. Doyle, and F.W. Sexton, "Nuclear Microprobe Imaging of Single Event Upset," IEEE Trans Nucl. Sci.NS-39, 1, 7 (1992).

[3] B.L. Doyle, K.M. Horn, D.S. Walsh, and F.W. Sexton, "Single Event Upset Imaging with a Nuclear Microprobe," Nucl. Instru. and Methods in Phys. Res. B64, 313 (1992).

[4] K.M. Horn, B.L. Doyle, F.W. Sexton, J.S. Laird, A. Saint, M. Cholewa, and G.J.F. Legge, "Ion Beam Induced Charge Collection (IBICC) Microscopy of ICs: Relation to Single Event Upset (SEU)," Nucl. Instru. and Methods in Phys. Res. B77, 355 (1993) .

[5] F.W. Sexton, K.M. Horn, B.L. Doyle, J.S. Laird, M. Cholewa, A. Saint, and G.J.F. Legge, "Ion-BeamInduced Charge-Collection (IBICC) Imaging of CMOS ICs," Nucl. Instru. and Methods in Phys. Res. B79, 436 (1993).

[6] M.B.H. Breese, G.W. Grime, and F. Watt, "Microcircuit Imaging Using Ion Beam Induced Current," Oxford Report OUNP-91-33, 1991.

[7] P.J. McNulty, "Track Structure Effects at p-n Junctions in Microelectronic Circuits," Nucl. Tracks Radiat. Meas. 16, 197 (1989).

[8] P.J. McNulty, W. G. Abdel-Kader, and J. E. Lynch, "Modeling Charge Collection and Single Event Upsets in Microelectronics," Nucl. Instru. and Methods in Phys. Res. B61 , 52 (1991).

[9] K.H. Lee, J.C. Desko, R.A. Kohler, C.W. Lawrence, W.J. Nagy, J.A. Shimer, S.D. Steenwyk, R.E. Ander- 
son, and J.S. Fu, "Radiation Hard 1.0- $\mu \mathrm{m}$ CMOS Technology," IEEE Trans. Nucl. Sci. NS-34, 1460 (1987).

[10] A. R Knudson, A. B. Campbell, P. Shapiro, W. J. Stapor, E. A. Wolicki, E. L. Petersen, S. E. Diehl-Nagle, J. Hauser, and P. V. Dressendorfer, "Charge Collection in Multilayer Structures," IEEE Trans Nucl. Sci. NS-31, 1149 (1984).

[11] J. S. Fu, C. L. Axness, and H. T. Weaver, "Memory SEU Simulations Using. 2-D Transport Calculations," IEEE Elec. Dev. Lett. EDL-6 , 422 (1985).

[12] J. R. Hauser, S. E. Diehl-Nagle, A. R. Knudson, and A. B. Campbell, "Ion Track Shunt Effects in MultiJunction Structures," IEEE Trans Nucl. Sci. NS-32, 4115 (1985).

[13] A. R. Knudson, A. B. Campbell, J. R. Hauser, M. Jessee, W. J. Stapor, and P. Shapiro, "'Charge Transport by the Ion Shunt Effect," IEEE Trans Nucl. Sci. NS-33, 1560 (1986).

[14] TRIM version 90.05, J.F. Ziegler, and J.P. Biersack, Pergamon Press, (1990).

[15] T.R. Oldham and F.B. Mclean, "Charge-Collection Measurements for Heavy Ions Incident on n- and pType Silicon," IEEE Trans Nucl. Sci. NS-30, 4493 (1983).

[16] F.W. Sexton, W.T. Corbett, R.K. Treece, K.J. Hass, K.L. Hughes, C.L. Axness, G.L. Hash, M.R. Shaneyfelt, and T.F. Wunsch, "SEU Simulation and Testing of Resistor-Hardened D-Latches in the SA3300 Microprocessor," IEEE Trans Nucl. Sci. NS-38, 1521 (1991).

[17] H.T. Weaver, C.L. Axness, J.D. McBrayer, J.S. Browning, J.S. Fu, A. Ochoa, Jr., and R. Koga, "An SEU Tolerant Memory Cell Derived from Fundamental Studies of SEU Mechanisms in SRAM," IEEE Trans Nucl. Sci. NS-34, 1281 (1987). 